\title{
Insight into polymorphism of the ethosuximide (ETX)
}

\author{
Natalia Osiecka ${ }^{1}$ Ewa Juszyńska-Gałązka ${ }^{1}$ Zbigniew Galewski ${ }^{2} \cdot$ Teresa Jaworska-Gołąb $^{3} \cdot$ Aleksandra Deptuch $^{3}$. \\ Maria Massalska-Arodź ${ }^{1}$
}

Received: 13 November 2017 / Accepted: 25 February 2018/ Published online: 8 March 2018

(c) The Author(s) 2018. This article is an open access publication

\begin{abstract}
Rich polymorphism of ethosuximide compound (ETX) is described in detail using complementary thermal analysis methods. The paper shows as well the results of differential scanning calorimetry (DSC), of polarizing microscope observations (POM) and of X-ray diffraction (XRD) measurements taken using horizontal rotating capillary method. Molecular structure of ethosuximide favors appearance of a conformationally disordered (CONDIS) crystal phase CrI in its polymorphism. Ethosuximide is a good glass former, and glass of the CrI phase was observed even for $5{ }^{\circ} \mathrm{C} \mathrm{min}{ }^{-1}$ rate of cooling. Moreover, monotropic plastic crystal CrII phase was observed during heating above the CrI temperature range.
\end{abstract}

Keywords Ethosuximide $\cdot$ Polarizing microscopy $\cdot$ Phase transition · Thermooptical analysis $\cdot$ DSC $\cdot$ CONDIS crystal vitrification

\section{Introduction}

Macroscopically, matter can exist in solid, melt and gas states. The large amplitude motions as translations and rotations of molecules, internal rotational and/or conformational changes determine the differences between fluid (IL) and solid states. In fact, some solid-state phases are not fully ordered crystal and not all large amplitude motions are frozen there. In liquid crystal (LC) phases, molecules act as orientationally (and in some cases even positionally) ordered but they are mobile to various extents. In plastic crystal phase (ODIC), molecules exhibit orientational mobility and local rotational disorder, but their centers of masses are positionally ordered. In conformationally

Electronic supplementary material The online version of this article (https://doi.org/10.1007/s10973-018-7142-x) contains supplementary material, which is available to authorized users.

Natalia Osiecka

Natalia.Osiecka@ifj.edu.pl

1 The Henryk Niewodniczański Institute of Nuclear Physics, Polish Academy of Sciences, E. Radzikowskiego 152, 31-342 Kraków, Poland

2 Faculty of Chemistry, University of Wrocław, Joliot-Curie 14, 50-383 Wrocław, Poland

3 Institute of Physics, Jagiellonian University, S. Łojasiewicza 11, 30-348 Kraków, Poland disordered (CONDIS) crystal phase, molecules are on average positionally and orientationally ordered, but they have partial (or full) conformational freedom [1-3]. It is well known that any disordered phase may be supercooled to form glass, and in case of LC, ODIC and CONDIS phases partially ordered glassy phases are formed [4]. When a compound exists in various solid-state forms, the following important questions should be asked: $1 /$ what is their thermodynamic stability, 2/ what are thermodynamic conditions in which any transformation can occur, and 3/ how long it lasts to have new phase in equilibrium state. Answers for those questions are given by thermal analysis methods.

Studying solid-state polymorphism of pharmaceutical compounds is a crucial issue, as each polymorph may have different bioactivities. In amorphous state, pharmaceuticals are more advantageous in therapy, so to gain knowledge of complexity of phase diagram and its evolution during storage may help in usage of smaller dose of medicine. Ethosuximide or 3-ethyl-3-methylpyrrolidine-2,5-dione (ETX) is a well-known substance used in epilepsy disease treatment [5]. In the literature, one can find publications on its bioactivity studies [6,7], while there is less information about ETX polymorphism and physicochemical properties. Most of physicochemical parameters were obtained at room temperature [8-10]. In this paper, results of differential scanning calorimetry (DSC), polarizing microscope observation (POM) and TOA thermooptical analysis are 
presented. This approach allowed to show rich solid-state polymorphism of ETX compound. To define structure of ETX polymorphs, XRD method was applied.

Figure 1 illustrates chemical formula of ETX, which consists of ethyl chain, methyl groups and imide ring. Such molecular structure allows to anticipate that ETX compound may exhibit a conformationally disordered crystal (CONDIS) in its polymorphism. It is known that conformational flexibility causes a reduced crystallization tendency and favors glass transition [11].

As we are going to present, complementary methods should be used in polymorphism investigation even for the material of such simple molecule as ETX. DSC method is one of the most commonly used techniques allowing to estimate the thermodynamic functions of the phase transition and its temperature. Microscopic texture observations together with thermooptical analysis (TOA) help in identification of liquid-like and solid-like phases found. Usually, DSC results are in good agreement with TOA [12-14]. By DSC alone, it is difficult to detect phase transitions characterized by small changes of heat capacity. TOA is not only very sensitive to any changes of phase structure but it does not suffer from thermal relaxation behavior after cooling/heating, what permits using fast rates of temperature changes during experiment [15]. The XRD results show difference in detail of ETX crystal structures.

\section{Experimental}

ETX sample was purchased in Sigma-Aldrich Company and studied on cooling and heating the samples with various rates of temperature changes in $0.2-50{ }^{\circ} \mathrm{C} \mathrm{min}-1$ range.

Polarizing microscope textures were observed using Biolar PI polarized microscope (PZO Warsaw) with the scanning rates $5,10,20$ and $50{ }^{\circ} \mathrm{C} \mathrm{min}{ }^{-1}$. The temperature was stabilized by Linkam THM 600 silver heating/cooling stage and TMS 90 temperature controller. Substance was placed between two glass plates at the temperature above

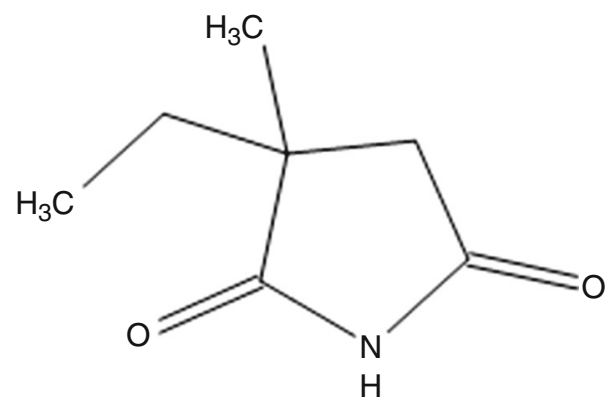

Fig. 1 Scheme of ethosuximide chemical formula melting point. Temperature was measured by platinum resistance thermometer with $0.1{ }^{\circ} \mathrm{C}$ accuracy.

Thermooptical analysis (TOA) was performed by TOApy program [15] based on digitalized images of ETX microscopic textures observed on cooling and heating experiments.

DSC measurements were taken using TA Instruments, Q2500. The mass of sample was equal to $13.56 \mathrm{mg}$. The sample was placed in aluminum TA Tzero pan and TA Tzero hermetic lid. During DSC experiment, the nitrogen purge was on the level 1.3 bar. The cooling/heating rate was $0.2,2,5,8,10,15$ and $20{ }^{\circ} \mathrm{C} \mathrm{min}{ }^{-1}$.

XRD measurements were taken in horizontal rotating capillaries made by borosilicate glass, with outside diameter $0.3 \mathrm{~mm}$ on Empyrean 2 (PANalytical) diffractometer with $\mathrm{CuK}_{\alpha}$ anode, parabolic mirror on the incident beam, slit for capillaries and PIXcel detector working in 1D scanning mode. The temperature was controlled with the help of Cryostream 700 Plus (Oxford Cryosystems). The data were collected in temperature range between $-90{ }^{\circ} \mathrm{C}$ and $50^{\circ} \mathrm{C}$ at several chosen temperature points during heating and subsequent cooling the sample. The XRD data were analyzed using XCell program from Material Studio software package. Fitting the XRD data was made using the Pawley refinement. The $R_{\mathrm{wp}}$ uncertainty parameter was on level $12 \%$ and $R_{\mathrm{p}}$ vary around $9 \%$.

\section{Results and discussion}

Results of POM observations and TOA analysis based on intensity changes of the light transformed through microscopic textures on cooling and heating the ETX samples are presented in Figs. 2-4. As it is shown in Fig. 2, during

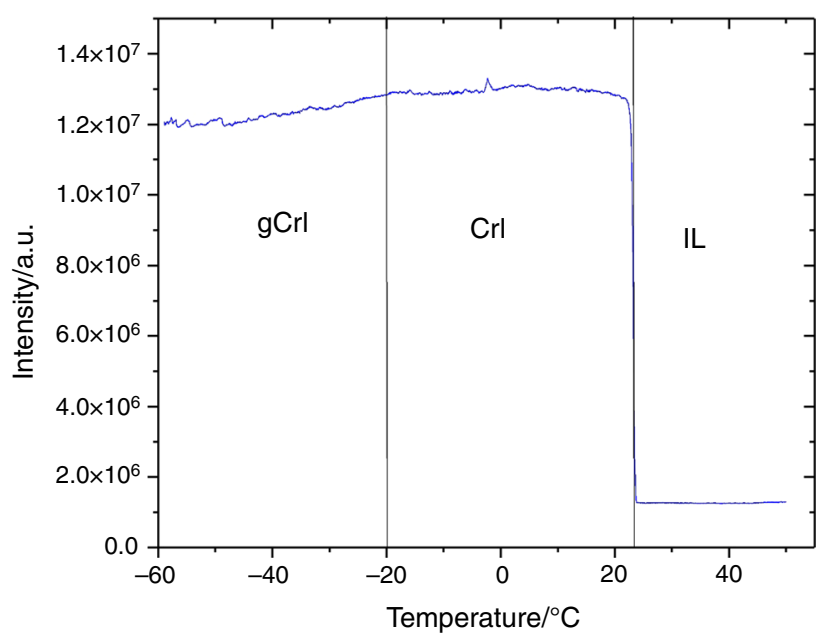

Fig. 2 TOA curve of light intensity transmitted through the microscopic textures of ETX sample during POM observed on cooling with rate $10{ }^{\circ} \mathrm{C} \mathrm{min}^{-1}$ 
cooling only one crystal phase and glass of that phase were observed. In IL phase, no texture was observed and due to dark image the line of low-light intensity in TOA plot is visible. At $25^{\circ} \mathrm{C}$, the light intensity jumps to higher value due to IL-CrI transition. In CrI it is on a stable level until $-20{ }^{\circ} \mathrm{C}$. Below this temperature cracks began to appear on CrI texture (see Fig. 3c) what is a well-known signature of glass transition identified in [16]. The tendency of vitrification is a characteristic feature of many ODIC phases. The observed glass is glass of plastic crystal CrI phase (gCrI).

During heating glass of CrI of the ETX compound, the first metastable CrI was identified at the TOA curve and then evidence of additional crystal CrII phase appearance was found. Softening of glass $\mathrm{gCrI}$ is observed until $18{ }^{\circ} \mathrm{C}$ as a slightly growing light intensity due to a process of cracks shrinkage. The CrI crystal phase is illustrated by a plateau in the TOA plot, and then, increase in intensity due to a solid-solid CrI-CrII transition is visible (see Fig. 4). Just below the isotropization point, a maximum is visible corresponding to a new crystal CrII phase with narrow temperature range of $48{ }^{\circ} \mathrm{C}-50{ }^{\circ} \mathrm{C}$.

Comparing plots in Figs. 2 and 4, one can see that ETX compound exhibits tendency to supercooling IL and CrI phases, which we found to be dependent on the thermal history in POM observations. It was established that crystallization temperature decreases with increasing of the cooling rate. Moreover, for the sample cooled with slow temperature change rates (i.e., $2-0.2{ }^{\circ} \mathrm{C} \mathrm{min}^{-1}$ ) crystallization was observed after 1-2 min. For higher cooling rates (i.e., $\quad 10-50{ }^{\circ} \mathrm{C} \min ^{-1}$ ), crystallization was not

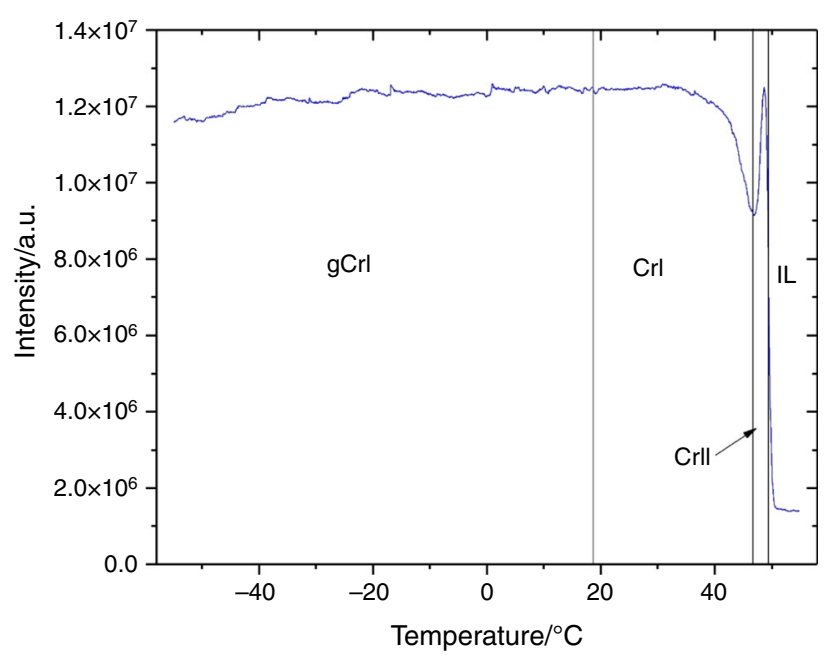

Fig. 4 TOA curve showing gCrI, CrI and CrII phases of ETX obtained on heating with rate $10{ }^{\circ} \mathrm{C} \mathrm{min}^{-1}$

observed at, for example, $30{ }^{\circ} \mathrm{C}$, when the waiting time was below $2 \mathrm{~h}$.

Figures 5-8 present results of DSC experiments. As one can see the unusual heat flow response to temperature decrease was detected (see inset in Fig. 5), while DSC plot transformed to heat flow vs time (see Fig. 6) illustrates a typical phase transition signature. The atypical DSC plot results from a phenomenon accompanying crystallization of supercooled IL phase [17] what will be explained in further part of this publication. All analyses were performed on heat flow DSC curves in function of time. The maxima corresponding to phase transitions were observed on cooling at $25.4{ }^{\circ} \mathrm{C}$ and during heating at $47.5{ }^{\circ} \mathrm{C}$ (with
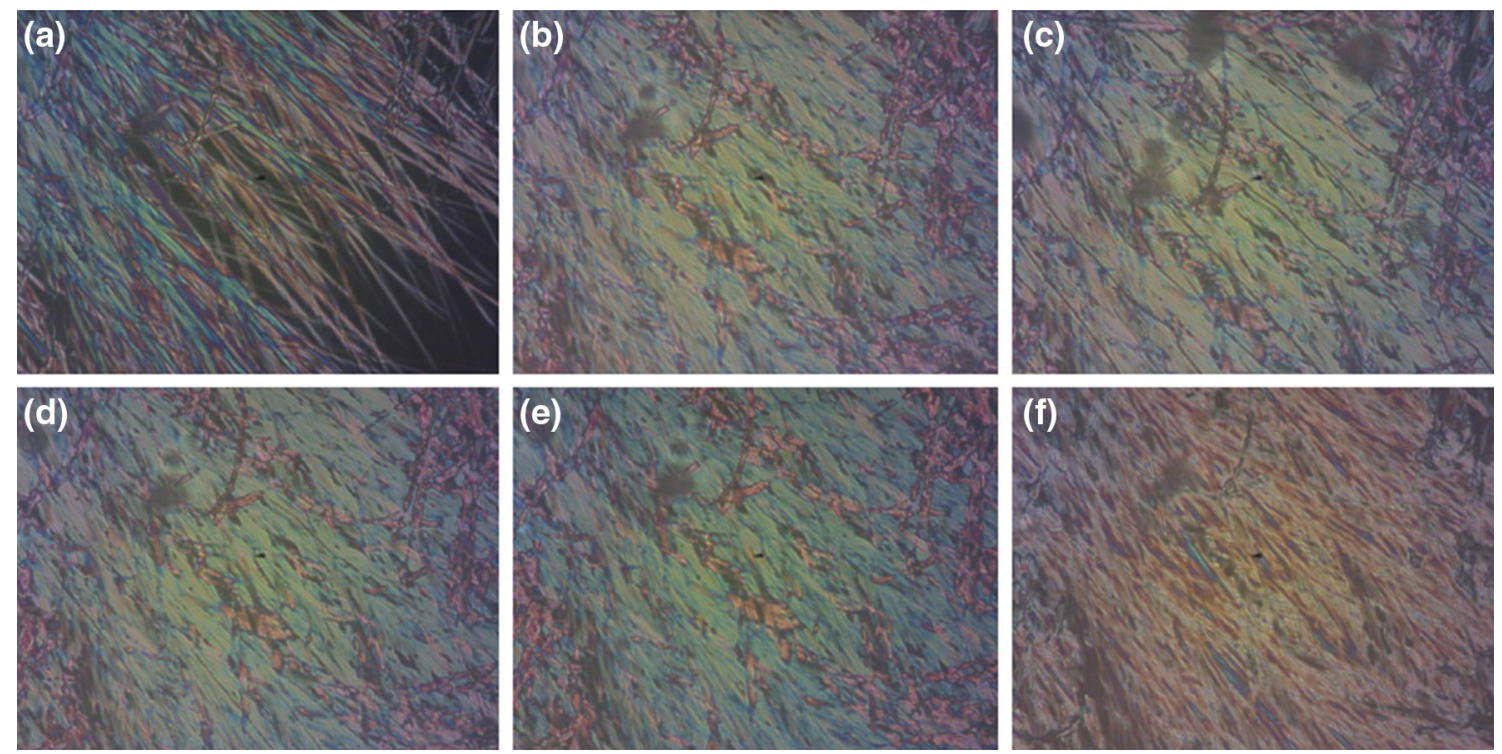

Fig. 3 Textures of ETX phases observed on cooling (upper images) and during heating (lower images) with rate of $10{ }^{\circ} \mathrm{C}$ min ${ }^{-1}: \mathbf{a}$ start of crystallization of $\mathrm{CrI}\left(25^{\circ} \mathrm{C}\right), \mathbf{b} \mathrm{CrI}\left(23^{\circ} \mathrm{C}\right)$, $\mathbf{c}$ glass of $\mathrm{CrI}\left(-30^{\circ} \mathrm{C}\right)$, d cracks shrinkage $\left(15^{\circ} \mathrm{C}\right)$, e crystal $\mathrm{CrI}$ phase $\left(40{ }^{\circ} \mathrm{C}\right)$ and $\mathbf{f} \mathrm{CrII}\left(48^{\circ} \mathrm{C}\right)$ 


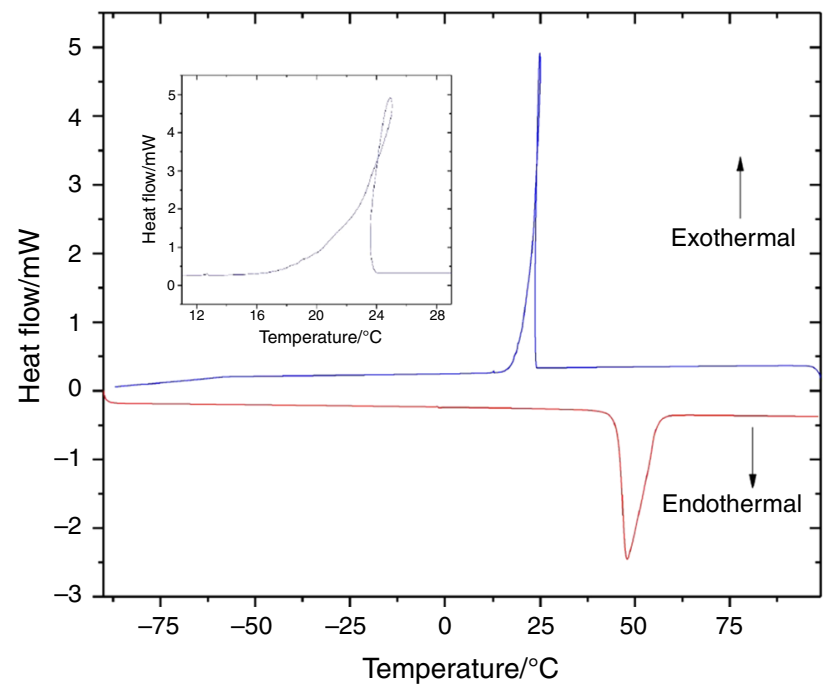

Fig. 5 DSC plot in function of temperature of ETX compound obtained during cooling (blue, upper line) and heating (red, lower line) with rate $10{ }^{\circ} \mathrm{C} \mathrm{min}^{-1}$. Inset illustrates cooling run in magnified temperature scale between 29 and $11{ }^{\circ} \mathrm{C}$

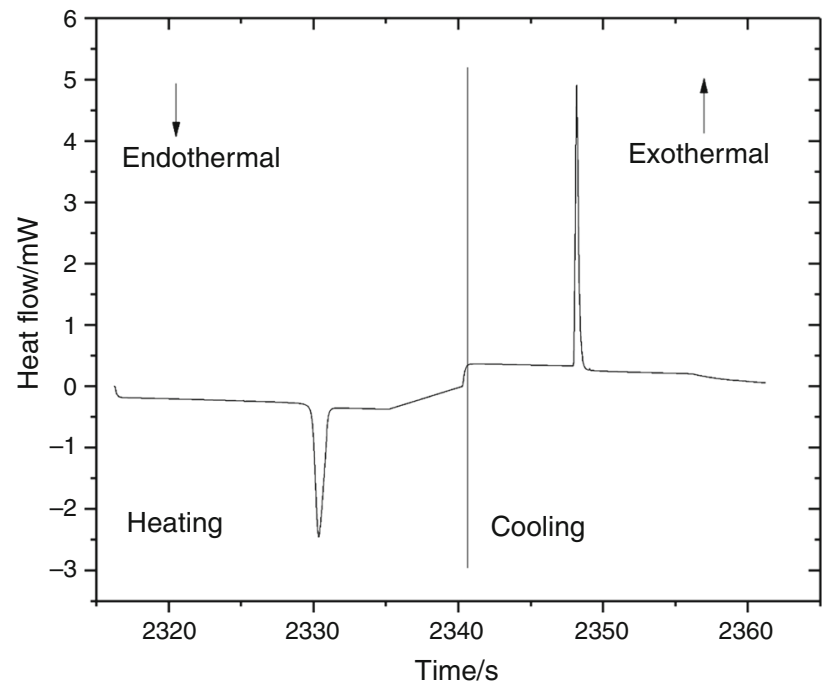

Fig. 6 DSC plot in function of time for ETX compound obtained during heating (sample cooled previously to $-90^{\circ} \mathrm{C}$ ) and on further cooling with rate of $10{ }^{\circ} \mathrm{C} \mathrm{min}-1$

rate $\pm 10{ }^{\circ} \mathrm{C} \min ^{-1}$ ). That observation seems to suggest the ETX has only one crystal phase. However, the value of full width at half maximum observed during heating is much higher than the value recorded during cooling. This information implies that during heating in fact two transitions may occur, at temperatures close to each other. Crystallization of CrI on cooling and CrI-CrII transition and melting of CrII on heating were already given by TOA analysis. The anomaly recorded during cooling corresponds to enthalpy change $\Delta H_{\text {cool }}=10.03 \mathrm{~kJ} \mathrm{~mol}^{-1}$, while for that observed during heating $\Delta H_{\text {heat }}=11.78 \mathrm{~kJ} \mathrm{~mol}^{-1}$. Those values are related to entropy change $\Delta S_{\text {cool }}=33.6 \mathrm{~J}$
$\mathrm{K}^{-1} \mathrm{~mol}^{-1}$ and $\Delta S_{\text {heat }}=36.7 \mathrm{~J} \mathrm{~K}^{-1} \mathrm{~mol}^{-1}$, respectively. Knowledge of magnitude of entropy change at phase transition is helpful to determine a type of solid phase which crystallizes/melts on cooling/heating run. In case of first order of phase transition, change of entropy $\Delta S_{\text {fusion }}$ is characterized by equation:

$\Delta S_{\text {fusion }}=\Delta S_{\mathrm{c}}+\Delta S_{\mathrm{o}}+\Delta S_{\mathrm{p}}$,

where $\Delta S_{\mathrm{c}}, \Delta S_{\mathrm{o}}$ and $\Delta S_{\mathrm{p}}$ correspond to entropy drop/jump at phase transition temperature given by freezing/activation of various degrees of freedom of molecules: conformational rotatable parts, orientational and positional, respectively. Usually $\Delta S_{\mathrm{p}}$ varies from 7 to $14 \mathrm{~J} \mathrm{~K}^{-1} \mathrm{~mol}^{-1}, \Delta S_{\mathrm{o}}$ occurs to have value in range $20-50 \mathrm{~J} \mathrm{~K}^{-1} \mathrm{~mol}^{-1}$, while $\Delta S_{\mathrm{c}}=\mathrm{n} \times(7-12) \mathrm{J} \mathrm{K}^{-1} \mathrm{~mol}^{-1}$, where $\mathrm{n}$ is a number of rotatable parts of the molecule [1, 18]. Based on this information, one may suggest that crystal phase observed during cooling is a CONDIS crystal due to zero value of $\Delta S_{\mathrm{c}}$ estimated. Registered entropy change $\Delta S_{\text {fusion }}=33.6$ $\pm 0.1 \mathrm{~J} \mathrm{~K}^{-1} \mathrm{~mol}^{-1}$ of IL-CrI phase transition seems to be the sum of $\Delta S_{o}=23 \pm 3 \mathrm{~J} \mathrm{~K}^{-1} \mathrm{~mol}^{-1}$ and $\Delta S_{p}=10 \pm$ $3 \mathrm{~J} \mathrm{~K}^{-1} \mathrm{~mol}^{-1}$. No contribution of $\Delta S_{\mathrm{c}}$ means that conformational disorder of ETX molecules in IL phase is probably the same as in the CONDIS CrI phase obtained. One can see that in case of heating experiment, values of entropies suggest that during heating rather two solid phases appeared, i.e., in addition to $\mathrm{CrI}$ identified on cooling an extra CrII was found only on heating ETX compound. The value of difference between $\Delta S_{\text {heat }}$ and $\Delta S_{\text {cool }}$ is equal around $3 \mathrm{~J} \mathrm{~K}^{-1} \mathrm{~mol}^{-1}$. It is similar as the value of difference that was found between smectic and nematic phases for a liquid crystal compound [18]. This observation suggests that differences between CrII and CrI crystal phases correspond to different orientational orders of ETX molecules in crystalline lattices. It seems that both crystalline phases are CONDIS crystals.

The peak separation corresponding to two phase transitions, which overlap to each other, may be observed, if the DSC experiment is performed with smaller temperature change. Figure 7 shows result of DSC plot obtained for ETX compound with heating range of $0.2{ }^{\circ} \mathrm{C} \mathrm{min}^{-1}$. As one can see the DSC peak is asymmetric and derivative of DSC plot points clearly two phase transitions near $48^{\circ} \mathrm{C}$.

The DSC experiment did not show evidence of glass transition, but the POM observation clearly shows cracks on the texture, which are signature of glass transition. During heating, cracks start to shrink until they disappear, what is a sign of glass softening. In Fig. 8, the temperature of glass transition $\mathrm{Tg}$ registered on cooling during POM observation is presented. Vitrification of phases with some degrees of disorder, i.e., of plastic crystals and conformationally disordered CONDIS crystals, is a well-known behavior. On changing the cooling rate from $50{ }^{\circ} \mathrm{C} \mathrm{min}-1$ 


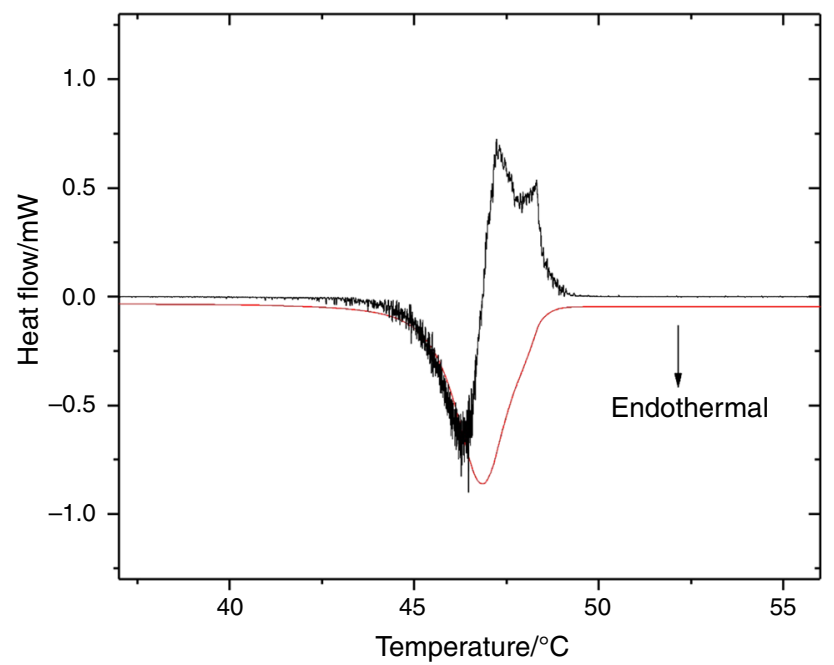

Fig. 7 DSC plot in function of temperature obtained with heating range $0.2{ }^{\circ} \mathrm{C} \mathrm{min}{ }^{-1}$ (red line) and its first derivative (black line)

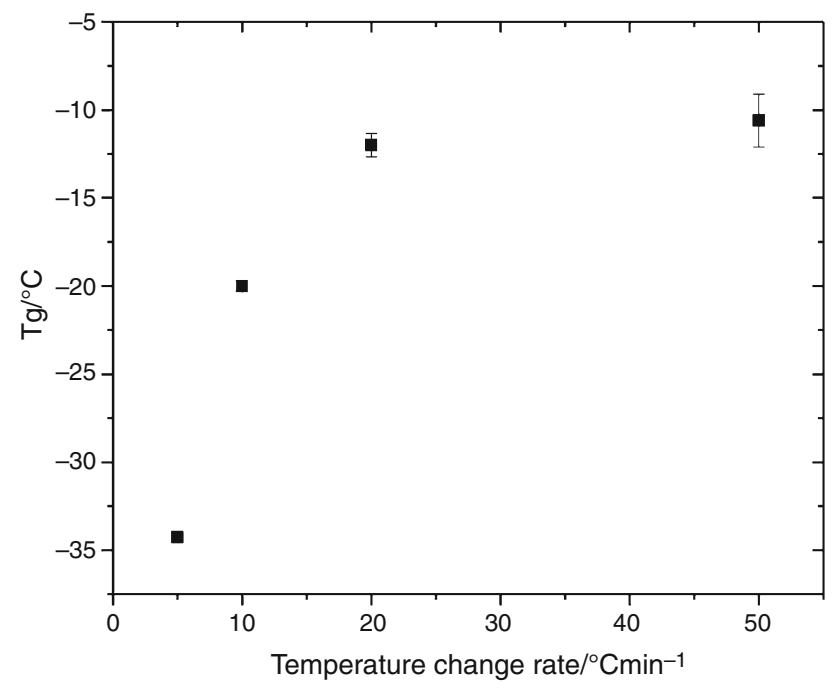

Fig. 8 Temperature of glass transition $(T \mathrm{~g})$ temperature obtained from POM observation with various cooling rates. The uncertainty of $\mathrm{Tg}$ temperature for experiment obtained for $5{ }^{\circ} \mathrm{C} \mathrm{min}^{-1}$ and for $10{ }^{\circ} \mathrm{C} \mathrm{min}^{-1}$ is smaller than the size of data point

to $5{ }^{\circ} \mathrm{C} \mathrm{min}^{-1}$, the $\mathrm{Tg}$ temperature shifts toward lower values (see Fig. 8). Usually, substances lose ability to vitrification if the cooling rate is lower than $8{ }^{\circ} \mathrm{C} \mathrm{min}-1$. ETX seems to be good glass former, as it shows good vitrification tendency even for $5{ }^{\circ} \mathrm{C} \mathrm{min}^{-1}$ cooling rate.

The thermodynamic conditions for equilibrium between phases and the directions of the possible phase transformations for an ETX compound at constant pressure are shown in schematic Gibbs free energy plot (Fig. 9). Since $\Delta G=G_{\mathrm{CrI}}-G_{\text {supercooled }}$ is $<0$, at some temperature spontaneous crystallization occurs in supercooled liquid (vertical blue arrow). Unusual DSC plot on cooling (see inset Fig. 5) points to conclusion that just above IL-CrI

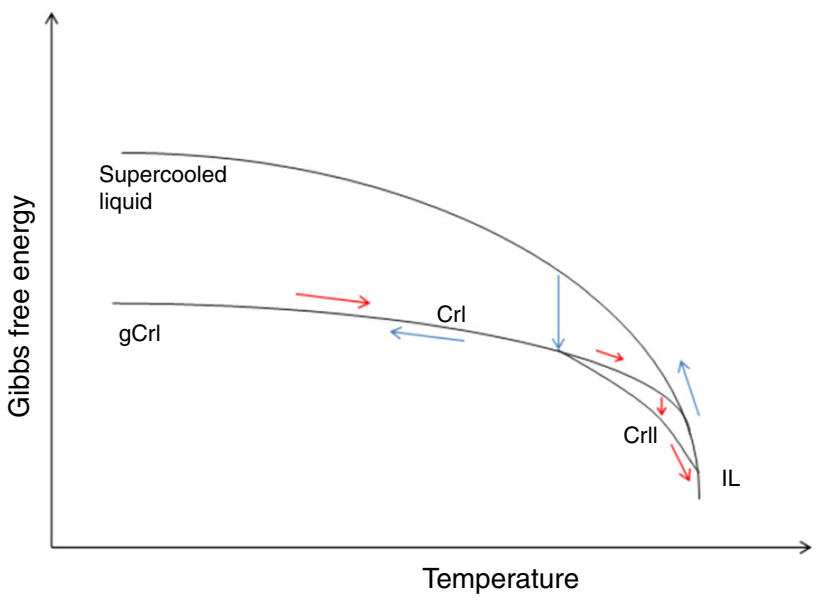

Fig. 9 Schematic free energy curves for ETX compound. Arrows in blue show changes on cooling, while arrows in red show changes on heating. Vertical arrows mean spontaneous transition from supercooled IL to CrI and from CrI to CrII. Vitrification and softening of $\mathrm{CrI}$ are not visible as well as weak effect of crystallization of CrII on cooling

transition temperature a small amount of the sample undergoes to CrII. This exudes a heat detected as the complex curve of heat flow vs $T$ observed on cooling. During further cooling vitrification of $\mathrm{CrI}$ occurs. While the sample is heated, softening of $\mathrm{gCrI}$ to $\mathrm{CrI}$ takes place. At the higher temperature range $G_{\mathrm{CrII}}-G_{\mathrm{CrI}}<0$ so at some temperature spontaneous transition of supercooled $\mathrm{CrI}$ to more stable CrII phase occurs (small vertical red arrow).

The results of XRD experiment are presented in Figs. 10-12. These data corroborate well polymorphism of ETX compound established above. Diffractograms presented in Fig. 10 show difference between data recorded at temperature $49{ }^{\circ} \mathrm{C}$ for $\mathrm{CrII}$ phase and at $20{ }^{\circ} \mathrm{C}$ for $\mathrm{CrI}$ phase. The temperature XRD patterns suggest that $\mathrm{CrI}$ crystallize in monoclinic P2 space group, while CrII in monoclinic $\mathrm{C} 2 / \mathrm{c}$. The temperature dependence of $\mathrm{a}, \mathrm{b}$ and $\mathrm{c}$

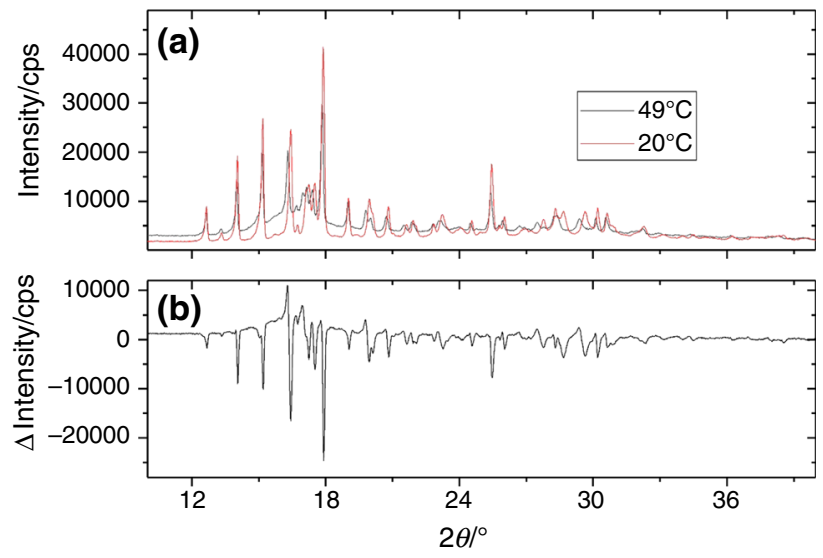

Fig. 10 a XRD pattern obtained during heating at temperature of $49^{\circ} \mathrm{C}$ (black line-CrII phase) and $20{ }^{\circ} \mathrm{C}$ (red line-Cr I phase) and b difference between those two patterns 

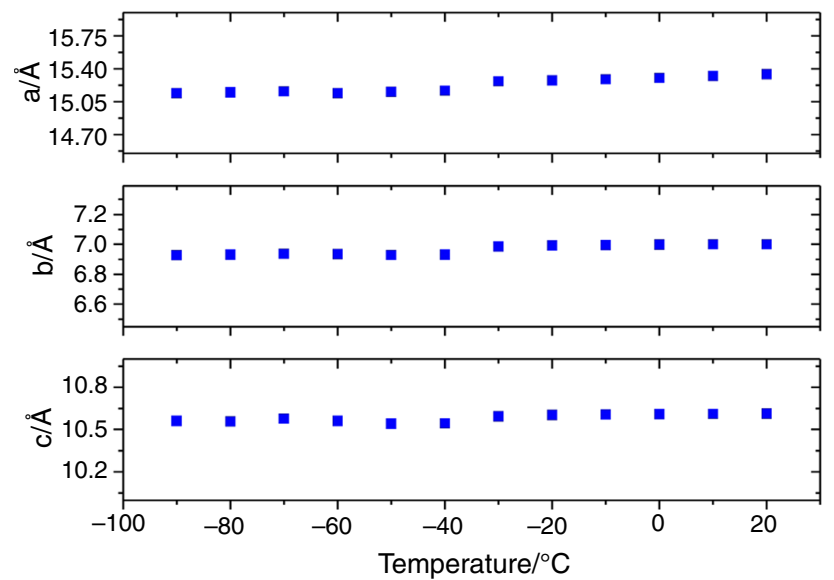

Fig. 11 Temperature dependence of unit cell parameters of CrI phase obtained during cooling. The uncertainty of data points is smaller than its size
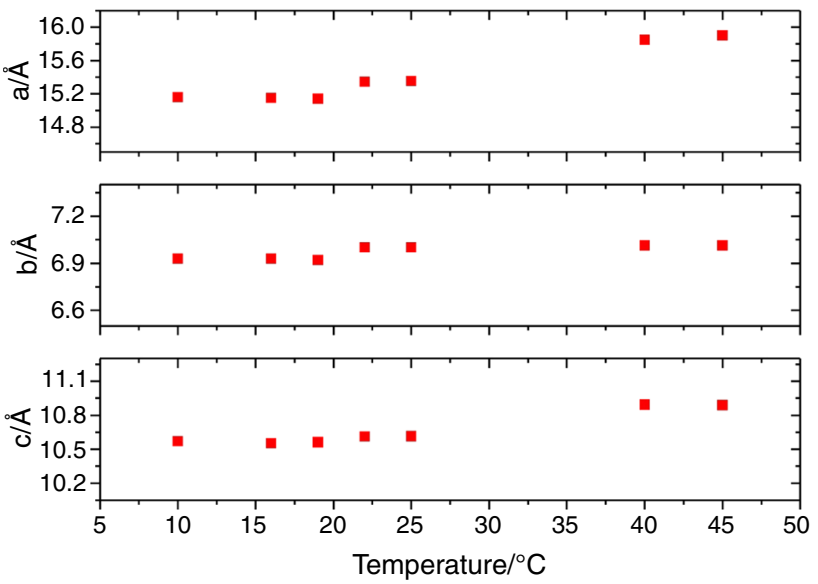

Fig. 12 Temperature dependence of unit cell parameters of CrI phase obtained during heating. The uncertainty of data points is smaller than its size

unit cell parameters of $\mathrm{CrI}$ phase is presented during cooling in Fig. 11 and on heating in Fig. 12, while the $\beta$ parameter varies from $92.67^{\circ}$ to $92.14^{\circ}$. A slight decrease in unit cell parameters around $-40{ }^{\circ} \mathrm{C}$ for cooling run and increase around $20{ }^{\circ} \mathrm{C}$ for heating run were found, which stay in good agreement with vitrification and glass softening temperatures obtained from thermal analysis methods. For the CrII unit cell parameters are $a=6.7641 \pm 0.0016, \quad b=28.0308 \pm 0.0039$, $c=21.4885 \pm 0.0061, \beta=98.4363 \pm 0.0023$.

\section{Conclusions}

ETX molecule compound occurs to show interesting solidstate polymorphism with the $\mathrm{CrI}, \mathrm{gCrI}$ and the CrII phases. POM observations and the TOA analysis have allowed to obtain isotropic CrI phase transition and vitrification of $\mathrm{CrI} /$ softening of glass of $\mathrm{CrI}$ and have clearly shown monotropic crystal CrII phase on heating. Based on the results of thermooptical methods, free energy Gibbs diagram of ETX has been proposed. The XRD measurements confirm the presence of two crystal phases and vitrification of CrI phase. Analysis of entropy changes at phase transitions given by DSC results points to conclusion that both crystalline phases are conformationally disordered. Thanks to analysis of derivative of heat flow vs temperature, the montropic CrII phase has been detected also by DSC method. The unusual DSC result found during crystallization of CrI on cooling was explained as accompanied by a weak process of crystallization of the CrII phase. The DSC experiment alone could not evidence of vitrification/softening phenomenon in ETX compound.

Open Access This article is distributed under the terms of the Creative Commons Attribution 4.0 International License (http://creative commons.org/licenses/by/4.0/), which permits unrestricted use, distribution, and reproduction in any medium, provided you give appropriate credit to the original author(s) and the source, provide a link to the Creative Commons license, and indicate if changes were made.

\section{References}

1. Wunderlich B. A classification of molecules, phases, and transitions as recognized by thermal analysis. Termochim. Acta. 1999:340-341:37-52.

2. Inaba A, Massalska-Arodź M, Suzuki H, Krawczyk J. (S)-4-(2methylbutyl)-4'-cyanobiphenyl $(5 * \mathrm{CB})$ glass former: are the crystalline polymorphs ordered? Mol Cryst Liq Cryst. 2011;540:102-10.

3. Juszyńska E, Jasiurkowska M, Massalska-Arodź M, Takajo D, Inaba A. Phase transition and structure studies of a liquid crystalline schiff-base compound (4O.8). Mol Cryst Liq Cryst. 2011;540:127-34

4. Rodriguez-Spong B, Price CP, Jayasankar A, Matzger AJ, Rodriguez-Hornedo N. Genaral principles of pharmaceutical solid polymorphism: a supermolecular perspective. Adv. Drug Deliv. Rev. 2004;56:241-74.

5. Sigler M, Strassburg HM, Boenigk HE. Effective and safe but forgotten: methsuximide in intractable epilepsies in childhood. Seizure. 2001;10:120-4.

6. He X, Zhong M, Zhang T, Wu W, Wu Z, Yang J, Xiao Y, Pan Y, Qiu G, Hu X. Synthesis and anticonvulsant activity of N-3-arylamide substituted 5,5-cyclopropanespirohydantoin derivatives. Eur J Med Chem. 2010;45:5870.

7. Kabra PM, Stafford BE, Marton LJ. Simultaneous measurement of phenobarbital, phenytoin, primidone, ethosuximide, and carbamazepine in serum by high-pressure liquid chromatography. Clin Chem. 1977;23:1284-8.

8. Krivoshein AV, Ordonez C, Khrustalev VN, Timofeeva TV. Distinct molecular structures and hydrogen bond patterns of a, a-diethyl-substituted cyclic imide, lactam, and acetamide derivatives in the crystalline phase. $J$ Mol Struct. 2016;1121:196-202. 
9. Lin PC, Su CS, Tang M, Chen YP. Micronization of ethosuximide using the rapid expansion of supercritical solution (RESS) process. J Supercrit Fluids. 2012;72:84-9.

10. Vijaya Chamundeeswari SP, Jebaseelan Samuel EJ, Sundaraganesan N. Quantum mechanical and spectroscopic (FT-IR, FTRaman, 13C, 1H and UV) investigations of antiepileptic drug Ethosuximide. Spectrochim Acta Part A. 2011;83:478-89.

11. Yu L, Reutzel-Edens SM, Mitchell CA. Crystallization and polymorphism of conformationally flexible molecules: problems, patterns, and strategies. Org Proc Res Dv. 2000;4:396-402.

12. Chachaj-Brekiesz A, Górska N, Osiecka N, Dynarowicz-Łątka P. Mesophases of non-conventional liquid crystalline molecules. J Therm Anal Calorim. 2016;126:689-97.

13. Chachaj-Brekiesz A, Górska N, Osiecka N, Makyła-Juzak K, Dynarowicz-Łątka P. Surface and liquid-crystalline properties of $\mathrm{F}_{\mathrm{m}} \mathrm{H}_{\mathrm{n}} \mathrm{F}_{\mathrm{m}}$ triblock semifluorinated n-alkanes. J Mat Sci Eng C. 2016;62:870-8.

14. Chachaj-Brekiesz A, Górska N, Osiecka N, Mikuli E, Dynarowicz-Łạtka P. Synthesis and thermal behavior of triblock semifluorinated n-alkanes. J Therm Anal Calorim. 2016;124:251-60.

15. Osiecka N, Galewski Z, Massalska-Arodź M. TOApy program for the thermooptical analysis of phase transitions. Termochim Acta. 2017;655:106-11.

16. Jasiurkowska-Delaport M, Juszyńska E, Kołek Ł, Krawczyk J, Massalska-Arodź M, Osiecka N, Rozwadowski T. Signatures of glass transition in partially ordered phases. Liq Cryst. 2013;40:1436-42.

17. Bamezai RK, Godlewska M, Massalska-Arodź M, Ściesiński J, Witko W. The adiabatic kalorymetry study of the polymorphism of solid 4,4'-di-n-butyloxyazoxybenzene. Phase Trans. 1990;27:113-9.

18. Horiuchi K, Yamamura Y, Pelka R, Sumita M, Yasuzuka S, Massalska-Arodź M, Saito K. Entropic contribution of flexible terminals to mesophase formation revealed by thermodynamic analysis of 4-alkyl-4'-isothiocyanatobiphenyl (nTCB). J Phys Chem B. 2010;114:4870-5. 